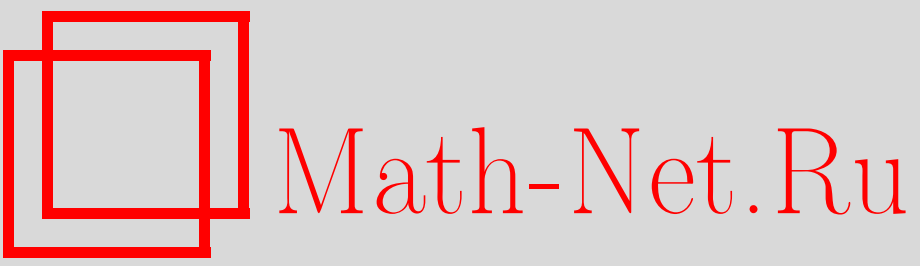

А. А. Джалилов, Гельдеровость сингулярных мер гомеоморфизмов окружности с одной точкой излома, ТМФ, 1999, том 121, номер 3, 355-366

DOI: https://doi.org/10.4213/tmf815

Использование Общероссийского математического портала Math-Net.Ru подразумевает, что вы прочитали и согласны с пользовательским соглашением

http://www.mathnet.ru/rus/agreement

Параметры загрузки:

IP : 3.82 .47 .9

26 апреля 2023 г., 14:57:09 


\section{ГЕЛЬДЕРОВОСТЬ СИНГУЛЯРНЫХ МЕР ГОМЕОМОРФИЗМОВ ОКРУЖНОСТИ С ОДНОЙ ТОЧКОЙ ИЗЛОМА}

Гомеоморфизмы окружности с особенностью типа излома имеют инвариантную меру, сингулярную относительно меры Лебега. Доказывается, что сингулярность инвариантной меры является гельдеровской.

\section{1. ВВЕДЕНИЕ}

В настояшей работе изучаются инвариантные меры гомеоморфизмов окружности с особенностями типа излома. Пусть функция $f(x)$ удовлетворяет следующим условиям [1]:

a) $f(x)$ - непрерывная, строго возрастаюшая функция на $\mathbb{R}^{1}$ и $0<f(0)<1$;

б) $f(x+1)=f(x)+1 \forall x \in \mathbb{R}^{1}$;

в) существует точка $x_{c} \in[0,1)$ такая, что $f(x) \in C^{2+\varepsilon}\left(\left[x_{c}, x_{c}+1\right]\right)$ при некотором $\varepsilon>0, f^{\prime}(x)>$ const $>0 \forall x \in\left[x_{c}, x_{c}+1\right]$;

г) односторонние производные $f^{\prime}\left(x_{c}-0\right)$ и $f^{\prime}\left(x_{c}+0\right)$ удовлетворяют соотношению

$$
\sqrt{\frac{f^{\prime}\left(x_{c}-0\right)}{f^{\prime}\left(x_{c}+0\right)}}=c \neq 1 .
$$

Всякий сохраняюший ориентацию гомеоморфизм окружности с одной точкой излома однозначно определяется функцией $f(x)$ и задается формулой

$$
T_{f} x=\{f(x)\}, \quad x \in S^{1}=[0,1),
$$

где $\{\cdot\}$ обозначает дробную часть числа. Гомеоморфизмы с одной точкой излома занимают промежуточное место между диффеоморфизмами, отвечаюшими $c=1$, и критическими отображениями окружности [1]. С одной стороны, для однопараметрических семейств гомеоморфизмов с особенностью типа излома множество значений параметра, отвечающих иррациональным числам вращения, имеет лебегову меру нуль, а сама

* Самаркандский государственный университет, Самарканд, Узбекистан. E-mail: info@mchmat.samuni.silk.org 
динамика характеризуется нетривиальными скейлингами. С другой стороны, как и в случае диффеоморфизмов окружности, ренормгрупповое поведение для подобных отображений выглядит достаточно просто. В перенормированных координатах итерации отображений асимптотически приближаются к дробно-линейным преобразованиям, зависящим лиш от двух параметров. Обозначим через $\rho=\rho(f)$ число вращения, отвечаюшее гомеоморфизму $T_{f}$. Всюду в дальнейшем будем предполагать, что число врашения $\rho$ иррационально. Хорошо известно, что в этом случае гомеоморфизм $T_{f}$ строго эргодический, т.е. обладает единственной нормированной инвариантной мерой $\nu$ [2]. Отсюда следует, что $T_{f}$ является эргодическим относительно меры $\nu$.

ТЕОрема 1.1. Пусть определяющая функиия $f(x)$ гомеоморфизма $T_{f}$ удовлетворяет условиям “а"- “г" и число вращения $\rho$ иррационально. Тогда $T_{f}$ является әргодическим относительно меры Лебега $\lambda$.

В отличие от типичных гладких диффеоморфизмов [3-7], инвариантные меры гомеоморфизмов с одним изломом сингулярны относительно меры Лебега [8]. Описание многих физических явлений может быть сведено к изучению статистических свойств сингулярных инвариантных мер $[9,10]$. Математически строгие результаты, относяшиеся к сингулярным мерам гомеоморфизмов окружности, были получены в работе [11] для критических гомеоморфизмов из класса $C^{3}$ с иррациональным числом врашения ограниченного типа. Последнее означает, что элементы разложения $\rho$ в непрерывную дробь равномерно ограничены.

Классическая теорема Данжуа легко переносится на случай гомеоморфизмов с особенностью типа излома [1]. Согласно этой теореме, если $\operatorname{var}_{\left[x_{c}, x_{c+1}\right]} \ln f^{\prime}(x)<\infty$, то сушествует гомеоморфизм окружности $\varphi$ такой, что

$$
\varphi\left(T_{f} x\right)=T_{\rho} \varphi(x), \quad x \in S^{1}
$$

где $T_{\rho} x=\{x+\rho\}-$ поворот окружности на угол $\rho$. С другой стороны, гомеоморфизм $\varphi$ можно выразить через инвариантную меру $\nu$ :

$$
\varphi(x)=\nu([0, x]), \quad x \in S^{1} .
$$

Определим две важные функции на окружности, характеризующие инвариантную меpy $\nu$,

$$
\underline{\tau}(x)=\varliminf_{\varepsilon \rightarrow 0} \frac{\ln |\varphi(x+\varepsilon)-\varphi(x)|}{\ln |\varepsilon|}, \quad \bar{\tau}(x)=\varlimsup_{\varepsilon \rightarrow 0} \frac{\ln |\varphi(x+\varepsilon)-\varphi(x)|}{\ln |\varepsilon|} .
$$

Функции $\underline{\tau}(x)$ и $\bar{\tau}(x)$ называются соответственно нижним и верхним показателями сингулярности для меры $\nu$.

ОПреДЕЛЕНИЕ 1.1. Сингулярная инвариантная мера $\mu$ называется гельдеровской, если сушествуют константы $C_{1}$ и $C_{2}, 0<C_{1}<C_{2}<1$, такие, что на множестве "полной” меры $\mu$ справедливы неравенства

$$
C_{1} \leqslant \underline{\tau}(x) \leqslant \bar{\tau}(x) \leqslant C_{2}
$$


Функции $\underline{\tau}(x)$ и $\bar{\tau}(x)$ - инвариантные функции относительно $T_{f}$. Отсюда, а также из эргодичности $T_{f}$ относительно мер $\nu$ и $\lambda$ следует, что обе эти функции являются почти постоянными по мере $\nu$ и по мере $\lambda$. Эти постоянные обозначим соответственно $\underline{\tau}(\nu), \bar{\tau}(\nu)$ и $\underline{\tau}(\lambda), \bar{\tau}(\lambda)$.

Сформулируем теперь основной результат работы.

ТЕОРема 1.2. Пусть определяющая функиия $f(x)$ гомеоморфизма $T_{f}$ удовлетворяет условиям “а”- “г”, а число вращения $\rho(f)$ иррациональное ограниченного типа. Тогда справедливы следующие неравенства:

1) $0<\underline{\tau}(\nu) \leqslant \bar{\tau}(\nu)<1$;

2) $1<\underline{\tau}(\lambda) \leqslant \bar{\tau}(\lambda)<+\infty$.

Из первого утверждения непосредственно следует гельдеровость инвариантной меры $\nu$.

Другой важной характеристикой сингулярной инвариантной меры $\nu$ является хаусдорфова размерность $H D(\nu)$ (точная нижняя грань размерностей множеств “полной" меры $\nu$ ).

ЛЕмма ФРОстмана. Пусть $\mu$-борелевская вероятностная мера на интервале и для почти всех $x$ (по мере $\mu)$

$$
\varliminf_{\varepsilon \rightarrow 0} \frac{\ln \mu([x, x+\varepsilon])}{\ln |\varepsilon|}=\chi .
$$

Тогда хаусдорфова размерность $H D(\mu)$ совпадает с $\chi$, m.е. $H D(\mu)=\chi$.

Из этой леммы следует, что $H D(\nu)=\underline{\tau}(\nu)$. Отсюда, используя первое утверждение теоремы 1.2 , получаем оценку для хаусдорфовой размерности

$$
0<H D(\nu)<1 .
$$

\section{2. ДИНАМИЧЕСКИЕ РАЗБИЕНИЯ ОКРУЖКОСТИ}

Рассмотрим сохраняюший ориентацию гомеоморфизм окружности $T_{f}$ с определяющей функцией $f(x)$, т.е.

$$
T_{f} x=\{f(x)\}, \quad x \in S^{1} .
$$

Пусть число врашения $\rho(f)$ иррационально и его разложение в непрерывную дробь имеет вид $\rho(f)=\left[k_{1}, k_{2}, \ldots, k_{n}, \ldots\right]$. Обозначим через $p_{n} / q_{n}=\left[k_{1}, k_{2}, \ldots, k_{n}\right], n \geqslant 1$, конечные дроби числа $\rho(f)$. Числа $p_{n}$ и $q_{n}$ удовлетворяют следующим рекуррентным соотношениям:

$$
\begin{aligned}
p_{n+1}=k_{n+1} p_{n}+p_{n-1}, & p_{0}=0, & p_{1}=1, \\
q_{n+1}=k_{n+1} q_{n}+q_{n-1}, & q_{0}=1, & q_{1}=k_{1} .
\end{aligned}
$$

Возьмем произвольную точку $x_{0} \in S^{1}$ и при помощи траектории этой точки построим последовательность разбиений окружности. Обозначим через $\Delta_{0}^{(n)} x_{0}$ замкнутую дугу, концами которой служат точки $x_{0}$ и $x_{q_{n}}=T_{f}^{q_{n}} x_{0}$. Заметим, что при нечетном $n$ точка $x_{q_{n}}$ лежит слева от $x_{0}$, а при четном $n$ - справа. Обозначим

$$
\Delta_{i}^{(n)}\left(x_{0}\right)=T_{f}^{i} \Delta_{0}^{(n)}\left(x_{0}\right), \quad i \geqslant 1 .
$$


Лемма 2.1 [12]. Точки $\left\{x_{i}, 0 \leqslant i<q_{n}+q_{n-1}\right\}$ разбивают окружность на непересекающиеся (за исключением кониевых точек) дуги: $\Delta_{i}^{(n-1)}\left(x_{0}\right), \quad 0 \leqslant i<q_{n}, u$ $\Delta_{j}^{(n)}\left(x_{0}\right), \quad 0 \leqslant j<q_{n-1}$.

Полученное разбиение обозначим через $\xi_{n}\left(x_{0}\right)$ и назовем динамическим разбиением $n$-го порядка.

Опишем теперь процесс перехода от $\xi_{n}\left(x_{0}\right)$ к $\xi_{n+1}\left(x_{0}\right)$. Все дуги $\Delta_{j}^{(n)}\left(x_{0}\right), 0 \leqslant j \leqslant$ $q_{n-1}-1$, сохраняются, а каждая дуга $\Delta_{i}^{(n-1)}\left(x_{0}\right), 0 \leqslant i \leqslant q_{n}-1$, разбивается на $k_{n+1}+1$ дуг:

$$
\Delta_{i}^{(n-1)}\left(x_{0}\right)=\Delta_{i}^{(n+1)}\left(x_{0}\right) \cup \bigcup_{s=0}^{k_{n+1}-1} \Delta_{i+q_{n-1}+s q_{n}}^{(n)}\left(x_{0}\right) .
$$

ЛЕмма ДАнЖУА [1]. Пусть в точке $x_{c} \in S^{1}$ существуют конечные односторонние производные $f^{\prime}\left(x_{c} \pm 0\right)$ :

$$
f^{\prime}\left(x_{c} \pm 0\right)>0, \quad f(x) \in C^{1}\left[x_{c}, x_{c}+1\right] \quad u \quad \operatorname{var}_{\left[x_{c}, x_{c}+1\right]} \ln f^{\prime}(x)=\bar{v}<\infty .
$$

Положим

$$
v=\bar{v}+\left|\ln f^{\prime}\left(x_{c}-0\right)-\ln f^{\prime}\left(x_{c}+0\right)\right| .
$$

Тогда для любого $y_{0}$ такого, что $y_{j} \neq x_{c}, \quad 0 \leqslant j \leqslant q_{k}-1, \quad k \in N$, справедливы неравенства

$$
e^{-v} \leqslant \prod_{j=0}^{q_{k}-1} f^{\prime}\left(y_{j}\right) \leqslant e^{v}
$$

Из леммы Данжуа следует, что длины дуг, составляющих динамическое разбиение $\xi_{n}\left(x_{0}\right)$, экспоненциально убывают. Более точно справедлива следующая лемма.

Лемма 2.2 [1]. Пусть выполнены условия леммы Данжуа. Тогда для любого $y_{0} \in S^{1}$

$$
\left|\Delta_{0}^{(n)}\left(y_{0}\right)\right| \leqslant \text { const } \lambda^{2 k}\left|\Delta_{0}^{(n-2 k)}\left(y_{0}\right)\right|, \quad 0 \leqslant 2 k<n,
$$

где $\lambda=\left(1+e^{-v}\right)^{-1 / 2}<1, a|\cdot|$ обозначает длину дуги.

В случае, когда число вращения $\rho$ является иррациональным типа константы, утверждение леммы 2.2 можно усилить.

ЛЕмма 2.3. Пусть выполнень условия леммы Данжуа и число вращения является иррачиональнымм типа константыл.

1. Тогда для любого $y_{0} \in S^{1}$ справедливы неравенства

$$
\text { const } \lambda_{1}^{2 k}\left|\Delta_{0}^{(n-2 k)}\left(y_{0}\right)\right| \leqslant\left|\Delta_{0}^{(n)}\left(y_{0}\right)\right| \leqslant \text { Const } \lambda^{2 k}\left|\Delta_{0}^{(n-2 k)}\left(y_{0}\right)\right|,
$$

где $\lambda_{1}, \quad 0<\lambda_{1}<\lambda$, не зависит от $n, k$ и $y_{0}$.

2. Пусть $\Delta_{s}^{(n)}\left(y_{0}\right) \in \xi_{n}\left(y_{0}\right), \Delta_{t}^{(n+1)}\left(y_{0}\right) \in \xi_{n+1}\left(y_{0}\right) u \Delta_{t}^{(n+1)}\left(y_{0}\right) \subset \Delta_{s}^{(n)}\left(y_{0}\right)$. Tozда существуют константы $C_{i}, 0<C_{i}<1, i=3,4$, такие, что,

$$
C_{3} \leqslant \frac{\left|\Delta_{t}^{(n+1)}\left(y_{0}\right)\right|}{\left|\Delta_{s}^{(n)}\left(y_{0}\right)\right|} \leqslant C_{4}
$$




\section{3. ДОКАЗАТЕЛЬСТВО ТЕОРЕМЫ 1.1}

В этом разделе мы докажем эргодичность $T_{f}$ относительно меры Лебега $\lambda$.

Предположим, что множество $A \subset S^{1}$ является инвариантным и $\lambda(A)>0$. Тогда $A$ имеет точку плотности $z_{0} \in S^{1}$. Зафиксируем $\varepsilon>0$. По определению точки плотности найдется такое $\delta>0$, что для любой дуги $[a, b]$, удовлетворяюшей условиям $z_{0} \in[a, b]$ и $[a, b] \subset\left(z_{0}-\delta, z_{0}+\delta\right)$, будет выполнено $\lambda(A \cap[a, b]) \geqslant(1-\varepsilon) \lambda[a, b]$ или иначе $\lambda(B \cap$ $[a, b])<\varepsilon \lambda[a, b]$, где $B=S^{1} \backslash A$. Обозначим $V_{0}^{(n)}\left(z_{0}\right)=\Delta_{0}^{(n-1)}\left(z_{0}\right) \cup \Delta_{0}^{(n)}\left(z_{0}\right)$. При достаточно больших $n$ имеем $V_{0}^{(n)}\left(z_{0}\right) \subset\left(z_{0}-\delta, z_{0}+\delta\right)$. Поэтому $\lambda\left(B \cap V_{0}^{(n)}\left(z_{0}\right)\right)<$ $\varepsilon \lambda\left(V_{0}^{(n)}\left(z_{0}\right)\right)$. Система дуг $\left\{V_{i}^{(n)}\left(z_{0}\right), i=\overline{0,\left(q_{n}-1\right)}\right\}$ покрывает окружность, и каждая точка окружности принадлежит не более чем двум дугам $V_{i}^{(n)}\left(z_{0}\right), i=\overline{0,\left(q_{n}-1\right)}$. Отсюда следует, что

$$
\sum_{i=0}^{q_{n}-1}\left|V_{i}^{(n)}\left(z_{0}\right)\right| \leqslant 2
$$

Фиксируем теперь $y_{0} \in V_{0}^{(n)}\left(z_{0}\right) \backslash\left\{T^{(m)} x_{c}, m \in Z^{\prime}\right\}$. Пусть $x_{c} \in V_{i}^{(n)}\left(z_{0}\right)$. Ясно, что

$$
\begin{aligned}
\frac{\frac{\left|V_{k}^{(n)}\left(z_{0}\right)\right|}{\left|V_{0}^{(n)}\left(z_{0}\right)\right|}}{\prod_{m=0}^{k-1} f^{\prime}\left(y_{i}\right)}= & \frac{1}{\left|V_{0}^{(n)}\left(z_{0}\right)\right|} \int \frac{\frac{d}{d x} f^{(i)}(x)}{\prod_{m=0}^{i-1} f^{\prime}\left(y_{m}\right)} d x \frac{1}{\left|V_{i}^{(n)}\left(z_{0}\right)\right|} \int_{V_{i}^{(n)}\left(z_{0}\right)} \frac{f^{\prime}(x)}{f^{\prime}\left(y_{i}\right)} d x \times \\
& \times \frac{1}{\left|V_{i+1}^{(n)}\left(z_{0}\right)\right|} \int_{V_{i+1}^{(n)}\left(z_{0}\right)} \frac{\frac{d}{d x} f^{(k-i)}(x)}{\prod_{m=i+1}^{k-1} f^{\prime}\left(y_{m}\right)} d x=I_{1} \times I_{2} \times I_{3} .
\end{aligned}
$$

Из условия $f^{\prime}(x) \geqslant$ const $>0 \forall x \in\left[x_{c}, x_{c}+1\right]$ следует, что

$$
\frac{m}{M} \leqslant I_{2} \leqslant \frac{M}{m}
$$

где $m=\min _{\left[x_{c}, x_{c}+1\right]} f^{\prime}(x), M=\max _{\left[x_{c}, x_{c}+1\right]} f^{\prime}(x)$. Теперь оценим $I_{1} \times I_{3}$. Пусть $x \in$ $V_{0}^{(n)}\left(z_{0}\right)$, тогда

$$
\prod_{\substack{m=0 \\ m \neq i}}^{k-1} \frac{f^{\prime}\left(T^{m} x\right)}{f^{\prime}\left(T^{m} y_{0}\right)}=\exp \left\{\sum_{\substack{m=0 \\ m \neq i}}^{k-1} \ln \left[1+\frac{f^{\prime \prime}\left(\theta_{m}\right)\left(T^{m} x-T^{m} y_{0}\right)}{f^{\prime}\left(T^{m} y_{0}\right)}\right]\right\} .
$$

Далее,

$$
\begin{aligned}
\left|\sum_{\substack{m=0 \\
m \neq i}}^{k-1} \ln \left[1+\frac{f^{\prime \prime}\left(\theta_{m}\right)\left(T^{m} x-T^{m} y_{0}\right)}{f^{\prime}\left(T^{m} y_{0}\right)}\right]\right| & \leqslant \text { const } \sum_{\substack{m=0 \\
m \neq i}}^{k-1}\left|T^{m} x-T^{m} y_{0}\right| \leqslant \\
& \leqslant \operatorname{const} \sum_{\substack{m=0 \\
m \neq i}}^{k-1}\left|V_{m}^{(n)}\left(z_{0}\right)\right| \leqslant \text { Const. }
\end{aligned}
$$


Используя (3.2) и (3.3), получаем

$$
\text { const } \leqslant I_{1} \times I_{3} \leqslant \text { Const }
$$

Из полученных выше оценок следует, что

$$
0<\text { const } \leqslant \frac{\frac{\left|V_{k}^{(n)}\left(z_{0}\right)\right|}{\left|V_{0}^{(n)}\left(z_{0}\right)\right|}}{\prod_{m=0}^{k-1} f^{\prime}\left(y_{m}\right)} \leqslant \text { Const }, \quad 0 \leqslant k \leqslant q_{n}-1
$$

Отсюда вытекает, что

$$
\begin{aligned}
\lambda\left(B \cap V_{k}^{(n)}\left(z_{0}\right)\right) & =\lambda\left(T^{k} B \cap T^{k} V_{0}^{(n)}\left(z_{0}\right)\right)=\lambda\left(T^{k}\left(B \cap V_{0}^{(n)}\left(z_{0}\right)\right)\right)= \\
& =\int_{B \cap V_{0}^{(n)}\left(z_{0}\right)} \frac{d}{d x} f^{(k)}(x) d x= \\
& =\prod_{m=0}^{k-1} f^{\prime}\left(y_{m}\right) \int_{B \cap V_{0}^{(n)}\left(z_{0}\right)} \frac{\frac{d}{d x} f^{(k)}(x)}{\prod_{m=0}^{k-1} f^{\prime}\left(y_{m}\right)} d x \leqslant \\
& \leqslant \text { const } \frac{\left|V_{k}^{(n)}\left(z_{0}\right)\right|}{\left|V_{0}^{(n)}\left(z_{0}\right)\right|} \lambda\left(B \cap V_{0}^{(n)}\left(z_{0}\right)\right) \leqslant \text { const } \varepsilon\left|V_{k}^{(n)}\left(z_{0}\right)\right| .
\end{aligned}
$$

Просуммируем по $k$ от 0 до $q_{n}-1$ :

$$
\lambda(B)=\sum_{k=0}^{q_{n}-1} \lambda\left(B \cap V_{k}^{(n)}\left(z_{0}\right)\right) \leqslant \text { const } \varepsilon \sum_{k=0}^{q_{n}-1}\left|V_{k}^{(n)}\left(z_{0}\right)\right| \leqslant \text { const } \varepsilon .
$$

В силу произвольности $\varepsilon$ имеем $\lambda(B)=0$. Отсюда следует, что $\lambda(A)=1$. Теорема 1.1 доказана.

\section{4. РАСХОЖДЕНИЕ МЕЖДУ РАЗБИЕНИЯМИ}

Здесь вводится величина, являющаяся мерой нелинейного поведения отображений с особенностями, и показывается, что гомеоморфизмы окружности с одной точкой излома лежат на некотором равномерном расстоянии от типичных диффеоморфизмов.

Пусть $I$ - замкнутая дуга единичной окружности. В дальнейшем будем рассматривать конечные или счетные разбиения $\xi$ дуги $I$ со следующими свойствами:

1. Каждый элемент $\omega$ разбиения $\xi$ есть замкнутая дуга, и любые две дуги $\xi$ могут пересекаться только в концевых точках.

2. Энтропия $H(\xi)$ конечна.

Определим вероятностную меру $\mu$ на $\xi$ по формуле

$$
\mu(X)=\sum_{\omega \in X} \frac{|\omega|}{|I|}
$$

где $X \subset \xi$ и $|\cdot|$ обозначает длину дуги. 
ОПРЕДЕЛЕНИЕ 4.1. Разбиения $\xi_{1}$ и $\xi_{2}$ дуг $I_{1}$ и $I_{2}$ называются изоморфныц.ми, если сушествует гомеоморфизм $\varphi: I_{1} \rightarrow I_{2}$, взаимно однозначно переводяший каждый элемент из $\xi_{1}$ в элемент из $\xi_{2}$.

Обозначим через $\mu_{i}, i=1,2$, вероятностные меры на $\xi_{i}, i=1,2$, определенные по формуле (4.1).

ОПРЕДЕЛЕнИЕ 4.2. Величиной расхождения между изоморфными разбиениями $\xi_{1}$ и $\xi_{2}$ называется число

$$
\sum_{\omega \in \xi_{1}}^{+} \ln \frac{\mu_{2}(h(\omega))}{\mu_{1}(\omega)} \mu(\omega)
$$

где $\sum^{+}$означает суммирование по всем таким $\omega$, при которых $\mu_{2}(h(\omega))>\mu_{1}(\omega)$. Значение суммы (4.2) обозначим через $\delta\left(\xi_{1}, \xi_{2}\right)$.

Из конечности $H\left(\xi_{i}\right), i=1,2$, следует, что ряд (4.2) сходится, т.е. $\delta\left(\xi_{1}, \xi_{2}\right)$ - конечное число.

Возьмем два экземпляра единичной окружности. Предположим, что на первой окружности действует гомеоморфизм $T_{f}$, а на второй - линейньй поворот $T_{\rho}$. Пусть начальная точка совпадает с точкой излома, т.е. $x_{0}=x_{c}$. Для каждого $n$ и $r(n, r \in \mathbb{N})$ обозначим через $\xi_{n r}$ и $\eta_{n r}$ динамические разбиения, полученные при помоши траекторий $x_{0}$ соответственно на первой и второй окружностях.

Рассмотрим дуги $\Delta^{(n r)}(f) \in \xi_{n r}$ и $\Delta^{(n r)}(\rho) \in \eta_{n r}, \quad \Delta^{(n r)}(\rho)=\varphi\left(\Delta^{(n r)}(f)\right)$. Ограничения $\xi_{(n+1) r}$ на $\Delta^{(n r)}(f)$ и $\eta_{(n+1) r}$ на $\Delta^{(n r)}(\rho)$ обозначим соответственно через $\xi_{(n+1) r}\left(\Delta^{(n r)}(f)\right)$ и $\eta_{(n+1) r}\left(\Delta^{(n r)}(\rho)\right)$. Сужение сопрягаюшего гомеоморфизма $\varphi$ на дугу $\Delta^{(n r)}(\rho)$ является изоморфизмом разбиений $\xi_{(n+1) r}\left(\Delta^{(n r)}(f)\right)$ и $\eta_{(n+1) r}\left(\Delta^{(n r)}(\rho)\right)$.

Tеорема 4.1. Существует некоторое натуральное число $r$ такое, что при достаточно больших п справедливо неравенство

$$
\delta\left(\xi_{(n+1) r}\left(\Delta^{(n r)}(f)\right), \eta_{(n+1) r}\left(\Delta^{(n r)}(\rho)\right)\right) \geqslant \text { const }>0 .
$$

ДоКАЗАТЕЛЬСТво. Для доказательства будем использовать ренормгрупповое поведение гомеоморфизма $T_{f}[1]$. Для определенности предположим, что $\Delta^{(n r)}(f)=$ $\Delta_{0}^{(n r-1)}(f)$ и $n r-$ четное число. Тогда

$$
\Delta_{0}^{(n r-1)}(f)=\left[x_{q_{n r-1}}, x_{0}\right], \quad \Delta_{0}^{(n r)}(f)=\left[x_{0}, x_{q_{n r}}\right] .
$$

В методе ренормгруппы важное место занимает изучение поведения следующих отображений при $n \rightarrow \infty$ :

$$
\begin{array}{r}
T_{f}^{q_{n r}}:\left[x_{q_{n r-1}}, x_{0}\right] \longrightarrow\left[x_{q_{n r-1}+q_{n r}}, x_{q_{n r}}\right], \\
T_{f}^{q_{n r-1}}:\left[x_{0}, x_{q_{n r}}\right] \longrightarrow\left[x_{q_{n r-1}}, x_{q_{n r-1}+q_{n r}}\right] .
\end{array}
$$


В окрестности $\left[x_{q_{n-1}}, x_{q_{n r}}\right]$ введем перенормированную переменную

$$
z=\frac{x-x_{0}}{x_{0}-x_{q_{n r-1}}}
$$

Очевидно, что $x_{0} \rightarrow 0$ и $x_{q_{n-1}} \rightarrow-1$ в новых координатах. Обозначим через $a_{n r}$ и $-b_{n r}$ перенормированные координаты соответствуюших точек $x_{q_{n r}}$ и $x_{q_{n-1}+q_{n r}}$, т.е.

$$
a_{n r}=\frac{x_{q_{n r}}-x_{0}}{x_{0}-x_{q_{n r-1}}}, \quad b_{n r}=\frac{x_{0}-x_{q_{n r-1}+q_{n r}}}{x_{0}-x_{q_{n r-1}}} .
$$

Положим

$$
M_{n r}=\exp \left\{\sum_{i=0}^{q_{n r}-1} \int_{x_{i+q_{n r-1}}}^{x_{i}} \frac{f^{\prime \prime}(y)}{2 f^{\prime}(y)} d y\right\}
$$

Заметим, что $0<-a_{n r} \leqslant b_{n r}<1$ и $\exp (-1 / 2 v) \leqslant M_{n r} \leqslant \exp (1 / 2 v)$. В новых координатах дугам $\left[x_{q_{n-1}}, x_{0}\right]$ и $\left[x_{0}, x_{q_{n r}}\right]$ соответствуют дуги $[-1,0]$ и $\left[0, a_{n r}\right]$, а $T_{f}^{q_{n r}}$ и $T_{f}^{q_{n r-1}}$ соответствуют следуюшие функции:

$$
\begin{array}{ll}
f_{n r}(z)=\frac{T_{f}^{q_{n r}}\left(x_{0}+z\left(x_{0}-x_{q_{n r-1}}\right)\right)-x_{0}}{x_{0}-x_{q_{n r-1}}}, & z \in[-1,0], \\
g_{n r}(z)=\frac{T_{f}^{q_{n r-1}}\left(x_{0}+z\left(x_{0}-x_{q_{n r-1}}\right)\right)-x_{0}}{x_{0}-x_{q_{n-1}}}, \quad z \in\left[0, a_{n r}\right] .
\end{array}
$$

Обозначим

$$
\begin{aligned}
& F_{n r}(z)=\frac{a_{n r}+\left(a_{n r}+b_{n r} M_{n r}\right) z}{1+\left(1-M_{n r}\right) z} \\
& G_{n r}(z)=\frac{-a_{n r} c+\left(c-b_{n r} M_{n r}\right) z}{a_{n r} c+\left(M_{n r}-c\right) z} .
\end{aligned}
$$

В работе [1] доказано, что для всех $n \geqslant 1$

$$
\begin{aligned}
\left\|f_{n r}(z)-F_{n r}(z)\right\|_{C^{2}[-1,0]} & \leqslant \operatorname{const} \lambda^{n r \varepsilon} \\
\left\|g_{n r}(z)-G_{n r}(z)\right\|_{C^{2}\left[0, a_{n r}\right]} & \leqslant \operatorname{const} \lambda^{n r \varepsilon}
\end{aligned}
$$

Дробно-линейные функции $F_{n r}$ и $G_{n r}$ являются строго возрастаюшими, и, кроме того, существует некоторая константа $\chi<0$ такая, что $\max _{[-1,0]} F_{n r}^{\prime \prime}(z)$ и $\max _{\left[0, a_{n r}\right]} G_{n r}^{\prime \prime}(z)$ меньше $\chi$. 
ЗАмечАниЕ. Если рассмотреть ренормгрупповое поведение функций $T_{f}^{q_{n r}} x$ и $T_{f}^{q_{n r}-1} x$ в окрестности $\left[x_{i+q_{n r-1}}, x_{i+q_{n r}}\right]$, то окажется, что функция $f_{n r}(z)$ близка (в смысле нормы $\left.C^{2}\right)$ на дуге $\left[-1, d_{n r}\right]$ к одной, а на дуге $\left[d_{n r}, 0\right]$ к другой дробно-линейной функции $\left(d_{n r}-\right.$ перенормированная координата точки $\left.x_{i-q_{n r}}\right)$. В то же время функция $g_{n r}(z)$ близка к соответствуюшей дробно-линейной функции $G_{n r}(z)$. Все эти дробно-линейные функции являются строго возрастаюшими и строго выпуклыми.

Продолжим доказательство теоремы 4.1. Заметим, что для каждой дуги $[\alpha, \beta] \in$ $\xi_{(n+1) r}\left(\Delta_{0}^{n r-1}\right)$

$$
\mu([\alpha, \beta])=z(\beta)-z(\alpha),
$$

где $z(\alpha)$ и $z(\beta)$ - перенормированные координаты точек $\alpha$ и $\beta$.

Выберем $r$ настолько большим, что при этом можно найти три последовательных (по расположению) дуги $\omega_{1}, \omega_{2}, \omega_{3}$ разбиения $\xi_{(n+1) r}\left(\Delta_{0}^{(n r-1)}(f)\right)$ со следуюшими свойствами:

1) дуга $\omega_{1} \cup \omega_{2} \cup \omega_{3}$ примыкает к точке $x_{q_{n-1}}$;

$2)$ дуги $T_{f}^{q_{n r}} \omega_{i}, i=\overline{1,3}$, тоже принадлежат разбиению $\xi_{(n+1) r}\left(\Delta_{0}^{(n r-1)}(f)\right)$.

Заметим, что при фиксированном $r$ перенормированные длины дуг разбиения $\xi_{(n+1) r}\left(\Delta_{0}^{(n r-1)}(f)\right)$ равномерно отделены от нуля, т.е.

$$
\frac{|\omega|}{\left|\Delta_{0}^{(n r-1)}(f)\right|} \geqslant \text { const }>0 \quad \forall \omega \in \xi_{(n+1) r}\left(\Delta_{0}^{(n r-1)}(f)\right) .
$$

Используя (4.3), получаем, что

$$
\frac{\mu\left(T_{f}^{q_{n r}} \omega_{i}\right)}{\mu\left(\omega_{i}\right)}=F_{n r}{ }^{\prime}\left(\theta_{i}\right)+O\left(\lambda^{n r}\right), \quad i=\overline{1,3},
$$

где

$$
\theta_{i} \in \frac{1}{\left|\Delta_{0}^{(n r-1)}(f)\right|} \omega_{i}, \quad i=\overline{1,3} .
$$

Отсюда имеем

$$
\frac{\frac{\mu\left(T^{q_{n r}} \omega_{1}\right)}{\mu\left(\omega_{1}\right)}}{\frac{\mu\left(T^{q_{n r}} \omega_{3}\right)}{\mu\left(\omega_{3}\right)}}=\frac{F_{n r^{\prime}}\left(\theta_{1}\right)}{F_{n r^{\prime}}\left(\theta_{3}\right)}+O\left(\lambda^{n r}\right)=1-\frac{F_{n r^{\prime \prime}}\left(\theta_{2}\right)}{F_{n r^{\prime}}\left(\theta_{3}\right)}\left(\theta_{3}-\theta_{1}\right)+O\left(\lambda^{n r}\right),
$$

где $\theta_{2} \in\left(\theta_{1}, \theta_{3}\right)$. Учитывая неравенства $\max _{[-1,0]} F_{n r}{ }^{\prime \prime}(z)<\chi<0$ и

$$
\theta_{3}-\theta_{1} \geqslant \frac{\left|\omega_{2}\right|}{\left|\Delta_{0}^{(n r-1)}(f)\right|},
$$

получаем

$$
\frac{\frac{\mu\left(T_{f}^{q n r} \omega_{1}\right)}{\mu\left(\omega_{1}\right)}}{\frac{\mu\left(T_{f}^{q n r} \omega_{3}\right)}{\mu\left(\omega_{3}\right)}} \geqslant \text { const }>1 .
$$


Используя изометричность $T_{\rho}$, находим, что

$$
\begin{gathered}
\ln \left(\frac{\frac{\mu\left(T_{f}^{q_{n} r} \omega_{1}\right)}{\mu\left(\omega_{1}\right)}}{\frac{\mu\left(T_{f}^{q_{n}} \omega_{2}\right)}{\mu\left(\omega_{2}\right)}}\right)=\ln \frac{\mu\left(T_{f}^{q_{n r}} \omega_{1}\right)}{\mu\left(\varphi\left(T_{f}^{q_{n r}} \omega_{1}\right)\right)}+\ln \frac{\mu\left(\omega_{3}\right)}{\mu\left(\varphi\left(\omega_{3}\right)\right)}+ \\
\quad+\ln \frac{\mu\left(\varphi\left(\omega_{1}\right)\right)}{\mu\left(\omega_{1}\right)}+\ln \frac{\mu\left(\varphi\left(T_{f}^{q_{n r}} \omega_{3}\right)\right)}{\mu\left(T^{q_{n r}} \omega_{3}\right)}>\text { const }>0 .
\end{gathered}
$$

Отсюда, а также из равномерной ограниченности вероятностей $\mu(\omega), \quad \omega \in$ $\xi_{(n+1) r}\left(\Delta_{0}^{(n r-1)}(f)\right)$, легко следует утверждение теоремы 4.1. Теорема 4.1 доказана.

\section{5. ДОКАЗАТЕЛЬСТВО ТЕОРЕМЫ 1.2}

Теорема 1.2 с небольшими изменениями доказывается так же, как и аналогичное утверждение для критических гомеоморфизмов из класса $C^{3}[11]$.

Введем удобные с технической точки зрения новые показатели сингулярности. Положим

$$
\bar{\gamma}(x)=\frac{1}{\underline{\tau}\left(\varphi^{-1}(x)\right)}, \quad \underline{\gamma}(x)=\frac{1}{\bar{\tau}\left(\varphi^{-1}(x)\right)} .
$$

Для доказательства первого утверждения теоремы 1.2 достаточно показать, что на множестве “полной" лебеговой меры имеют место неравенства

$$
K<\underline{\gamma}(x) \leqslant \bar{\gamma}(x)<\infty
$$

где $K>1$ не зависит от $x$.

Лемма 5.1. Существует некоторая константа $K_{1}>1$ такая, что на множестве "полной" лебеговой меры выполнено неравенство

$$
K_{1} \leqslant \bar{\gamma}(x)
$$

Для доказательства леммы 5.1 потребуется следующая лемма.

ЛЕмма 5.2 [11]. Пусть гомеоморфизм $h$ дуги $I$ на $J$ является изоморфизмом разбиений $P(I), P(J)$ и удовлетворяет следующему условию:

$$
\sum_{\omega \in P(I)} \frac{|\ln \mu(h(\omega))|}{|\ln | J||}|\ln \mu(\omega)| \mu(\omega) \leqslant \operatorname{const} \delta^{2}(P(I), P(J)) .
$$

Если для некоторой положительной константы $K_{2}$ выполняется неравенство

$$
\frac{\ln |J|}{\ln |I|} \leqslant \min \left\{2,1+\frac{K_{2}}{H(P(I))} \delta^{2}(P(I), P(J))\right\},
$$

то существует константа $K_{3}>0$ такая, что

$$
\sum_{\omega \in P(I)} \frac{\ln |h(\omega)|}{\ln |\omega|} \mu(\omega)>\frac{\ln |J|}{\ln |I|}\left(1+K_{3} \frac{\delta^{2}(P(I), P(J))}{\ln |I|}\right) .
$$


ДОКАЗАТЕЛЬСТвО ЛЕмМЫ 5.1. Рассмотрим последовательности динамических разбиений $\left\{\eta_{n r}, n \geqslant 1\right\}$ и $\left\{\xi_{n r}, n \geqslant 1\right\}$. Пусть $\Delta^{(n r)}(\rho) \in \eta_{n r}$ и $\Delta^{(n r)}(f)=$ $\varphi^{-1}\left(\Delta^{(n r)}(\rho)\right) \in \xi_{n r}$. $\quad$ Рассмотрим лемму 5.2 в применении к разбиениям $\eta_{(n+1) r}\left(\Delta^{(n r)}(\rho)\right)$ и $\xi_{(n+1) r}\left(\Delta^{(n r)}(f)\right)$. Поскольку $\rho(f)$ - иррациональное число типа константы, с помощью леммы 2.3 получаем:

1) $\ln \mu\left(\varphi^{-1}(\omega)\right)$ и $\ln \mu(\omega)$ равномерно ограничены снизу и сверху;

2) для всех $n \geqslant 1$ имеет место неравенство

$$
\ln \left|\Delta^{(n r)}(f)\right| \leqslant \operatorname{const} \frac{1}{n} .
$$

В соответствии с теоремой 4.1 имеем

$$
\delta\left(\eta_{(n+1) r}\left(\Delta^{(n r)}(\rho)\right), \xi_{(n+1) r}\left(\Delta^{(n r)}(f)\right)\right) \geqslant \text { const }>0 .
$$

Из вышесказанного следует, что сушествует $n_{0}=n_{0}(f)$ такое, что при $n \geqslant n_{0}$ выполняется (5.1).

ОПРЕДЕЛЕнИЕ 5.1. Отрезок $\Delta^{(n r)}(\rho)$ разбиения $\eta_{n r}$ назовем "хорошим", если он удовлетворяет условию (5.2) леммы 5.2. В противном случае он называется "плохим" и обозначается через $I^{n r}$.

Используя определение 5.1, предложим схему выбора элементов $\eta_{n r}, n \geqslant n_{0}$. Берем все "хорошие" элементы. Если $\eta_{n r}$ содержит "плохой" элемент $I^{n r}$, то переходим к разбиению $\eta_{(n+1) r}\left(I^{n r}\right)$ и т.д.

Обозначим через $M$ подмножество окружности $S^{1}$, каждая точка которого бесконечно много раз покрывается "плохими" дугами разбиений $\eta_{n r}, n \geqslant n_{0}$. Покажем, что $\lambda(M)=0$. Заметим сначала, что для каждого $n$ объединение всех "плохих" дуг $I^{n r}$ из $\eta_{n r}$ покрывает $M$. Используя лемму 2.3 , получаем для всех $m \geqslant 1$

$$
\text { const } \frac{1}{m} \leqslant \max _{I^{m r} \in \eta_{m r}} \frac{\ln \varphi^{-1}\left(I^{m r}\right)}{\ln ^{2}\left|I^{m r}\right|} \leqslant \text { const } \frac{1}{m} .
$$

Повторно используя лемму 5.2, а также неравенства (5.3) и (5.4), получаем, что

$$
\begin{aligned}
\sum_{I^{n r} \in \eta_{n r}} \frac{\ln \left|\varphi^{-1}\left(I^{n r}\right)\right|}{\ln \left|I^{n r}\right|}\left|I^{n r}\right|> & \sum_{I^{n_{0} r} \in \eta_{n r}} \frac{\ln \left|\varphi^{-1}\left(I^{n_{0} r}\right)\right|}{\ln \left|I^{n_{0} r}\right|}\left|I^{n_{0} r}\right|+K_{4} \lambda(M) \sum_{m=n_{0}}^{n-1} \frac{1}{m}- \\
& -\sum_{m=n_{0}} \sum_{\omega_{g}^{m r} \in \eta_{m r}} \frac{\ln \left|\varphi^{-1}\left(\omega_{g}^{m r}\right)\right|}{\ln \left|\omega_{g}^{m r}\right|}\left|\omega_{g}^{m r}\right|
\end{aligned}
$$

где через $\omega_{g}^{m r}$ обозначена "хорошая" дуга разбиения $\eta_{m r}$ и $K_{4}>0$ не зависит от $n$. Легко видеть, что все суммы (5.5), кроме второй в правой части, ограничены сверху. Если $\lambda(M)>0$, то правая часть (5.5) при $n \rightarrow+\infty$ стремится $\mathrm{x}+\infty$, что невозможно. Значит, $\lambda(M)=0$. Следовательно, лебегова мера всех "хороших" дуг равна 1. 
Полученные "хорошие" дуги мы назовем "хорошими” дугами первого поколения. Далее, применяя к каждой “хорошей” дуге первого поколения вышеуказанную процедуру выбора, получаем “хорошие” дуги второго поколения и т.д. В итоге получим систему "хороших" дуг разных поколений. Из конструкции видно, что каждая "хорошая" дуга $n$-го поколения мельче, чем любой элемент разбиения $\eta_{(n-1) r}$. Возьмем $x \in S^{1}$ и обозначим через $G_{x}^{n r}$ "хорошую" дугу из $\eta_{n r}$, содержащую точку $x$. Обозначим через $B$ множество, состояшее из таких точек окружности, которые бесконечно много раз покрываются "хорошими" дугами. Ясно, что $\lambda(B)=1$. Если $x \in B$, то $x \in G_{x}^{n_{i} r}$, $n_{1}<n_{2}<\cdots<n_{i}<n_{i+1}<\cdots$ и

$$
\bar{\gamma}(x) \geqslant \min \left\{2,1+\frac{K_{5}}{H\left(\eta_{\left(n_{i}+1\right) r}\left(G_{x}^{n_{i} r}\right)\right)}\right\},
$$

где $K_{5}>0$ на зависит от $n$. Учитывая, что энтропия $H\left(\eta_{\left(n_{i}+1\right) r}\left(G_{x}^{n_{i} r}\right)\right)$ равномерно ограничена сверху, получим

$$
\bar{\gamma}(x) \geqslant 1+K_{6}
$$

где $K_{6}>0$ не зависит от $x$. Лемма 5.1 доказана.

Так же, как и в работе [11], на основании леммы 5.1 можно показать справедливость неравенства

$$
K_{7}<\underline{\gamma}(x)
$$

на множестве "полной" лебеговой меры, где $K_{7}>1$ не зависит от $x$. Тем самым первое утверждение теоремы 1.2 доказано. Повторяя доказательство первого утверждения, поменяв ролями $T_{\rho}$ и $T_{f}$, получаем второе утверждение теоремы 1.2 . Теорема 1.2 доказана.

Благодарности. Автор выражает свою глубокую благодарность Я.Г. Синаю, К. М. Ханину и Дж. Грачеку за полезные обсуждения.

\section{Список литературы}

[1] K. M. Khanin, E. B. Vul. Adv. Sov. Math. 1991. V. 3. P. 57

[2] И. П. Корнфельд, Я.Г. Синай, С. В. Фомин. Эргодическая теория. М.: Наука, 1980.

[3] J. C. Yoccoz. Ann. Sci. Ec. Norm. Sup. Ser. 4. 1984. V. 17. P. 333.

[4] K. M. Khanin, Ya. G. Sinai. Commun. Math. Phys. 1987. V. 112. P. 89

[5] Я.Г. Синай, К. М. Ханин. УМН. 1989. Т. 44. № 1. С. 57.

[6] Y. Katznelson, D. Ornstein. Ergodic Theory and Dyn. Systems. 1989. V. 9. № 4. P. 643.

[7] J. Stark. Nonlinearity. 1988. V. 1. P. 541.

[8] А. А. Джсалилов, К. М. Ханин. Функц. анализ и его прилож. 1998. Т. 32. № 3. С. 11.

[9] A. Chhabra, R. V. Jensen. Phys. Rev. Lett. 1989. V. 62. P. 1327.

[10] T.S. Halsey, M. N. Jensen, L.P. Kadanoff, I. Procaccia, B.I. Shraiman. Phys. Rev. A. 1986. V. 33. P. 1141.

[11] J. Graczyk, G. Swiatek. Commun. Math. Phys. 1993. V. 157. № 2. P. 213

[12] Я. Г. Синай. Современные проблемы эргодической теории. М.: Изд. фирма "Физико-математическая литература", 1995. 